\title{
Some Studies on Fuzzy Generalized Open Sets
}

\author{
${ }^{1}$ Basayya B. Mathad, ${ }^{2}$ Rakesh Umadi \\ ${ }^{1}$ Department of Mathematics \\ S.G. Balekundri Institute of Technology \\ Belagavi-590010, Karnataka, India \\ bbmath.mathad@gmail.com \\ ${ }^{2}$ Department of Mathematics \\ S.G. Balekundri Institute of Technology \\ Belagavi-590010, Karnataka, India \\ rakeshumadi2@gmail.com
}

Article History: Received: 11 January 2021; Accepted: 27 February 2021; Published online: 5 April 2021

\begin{abstract}
In this paper, we introduce important fuzzy generalized open sets namely fuzzy Generalized Regular open (briefly, fuzzy GR-open) sets. This new class shows stronger properties in fuzzy topological spaces and also, we investigate fuzzy GR-neighbourhoods and fuzzy GRcontinuity properties.
\end{abstract}

Keywords- fuzzy GR-open sets, fuzzy GR-neighbourhood, fuzzy GR-interior, fuzzy GR-closure, fuzzy GR-continuous maps.

\section{INTRODUCTION}

Fuzzy regular open sets have been introduced and investigated by Azad K.K. [2]. Balasubramanian, G. et al.[3], Azad K.K. [2], Zahren A.N. [5] and Saziye Yuksel et al. [4] have discussed fuzzy g-closed sets, fuzzy semiopen sets, fuzzy regular semiopen sets and fuzzy $\delta$-open sets respectively. Balasubramanian introduced the concept of fuzzy g-closure and fuzzy ginterior.

We introduce fuzzy Generalized Regular open sets in topological spaces, which mainly exists between the class of fuzzy regular open sets and the class of fuzzy open sets. Also, we discuss the new system of neighbourhoods called fuzzy Generalized Regular neighourhoods and fuzzy Generalized Regular continuous properties.

\section{PRELIMINARIES}

Throughout this paper $(\mathrm{X}, \tau),(\mathrm{Y}, \sigma)$ and $(\mathrm{Z}, \eta)$ or simply $\mathrm{X}, \quad \mathrm{Y}$ and $\mathrm{Z}$ always denote fuzzy topological spaces on which no separation axioms are assumed unless explicitly stated. Fint $(\gamma), \quad \operatorname{Fcl}(\gamma)$ denote the interior of $\gamma$, closure of $\gamma$ in $X$ respectively. $1-\gamma$ or $\gamma^{\mathrm{c}}$ denotes the complement of $\gamma$ in $\mathrm{X}$. We recall the following definitions and results.

Definition 2.1. A subset $\gamma$ of a topological space $X$ is called

(1) Fuzzy regular open [2], if $\gamma=\operatorname{int}(\operatorname{cl}(\gamma))$ and fuzzy regular closed if $\operatorname{cl}(\operatorname{int}(\gamma))=\gamma$.

(2) Fuzzy semi open [2], if $\gamma \leq \operatorname{cl}(\operatorname{int}(\gamma))$ and fuzzy semi $\operatorname{closed}[2]$ if $\operatorname{int}(\operatorname{cl}(\gamma)) \leq \gamma$.

(3) Regular semi open set [5] if there is a fuzzy regular open set $\xi$ such that $\xi \leq \gamma \leq$ $\operatorname{cl}(\xi)$.

(4) $\pi$-open [1], if $\xi$ is a finite fuzzy union of fuzzy regular open sets. The complement of $\pi$-open set is called the $\pi$-closed set.

Definition 2.2. A subset $\gamma$ of a topological space $X$ is called

(1) Fuzzy g-closed [3] if $\operatorname{cl}(\gamma) \leq \xi$ whenever $\gamma \leq \xi$ and $\xi$ is fuzzy open in $\mathrm{X}$.

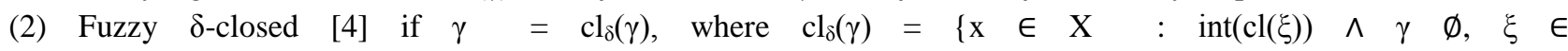
$\gamma\}$.

The complement of above all fuzzy closed sets are their respective fuzzy open sets in the same fuzzy topological space. 
Research Article

Definition 2.3. Let $X$ be any fuzzy topological space and $\gamma \leq X$, then the fuzzy g-closure[3] of is denoted by fuzzy $\mathrm{g}-\mathrm{cl}(\gamma) . \quad \gamma$ is the fuzzy intersection of all fuzzy g-closed sets in $\mathrm{X}$ containing $\gamma$. The fuzzy g-closure of $\gamma$.

Definition 2.4. Let $X$ be a fuzzy topological space and $\gamma \leq X$, then the fuzzy g-interior[3] of denoted by fuzzy $g$-int $(\gamma) . \quad \gamma$ is the fuzzy union of all fuzzy $g$-open sets in $X$ contained in $\gamma$. The fuzzy g-interior of $\gamma$ is

Lemma 2.1. Let $X$ be any topological space and $\gamma$ and $\xi$ are subsets of $X$. Then following properties holds

(1) $\operatorname{Fg}-\operatorname{cl}(\gamma \wedge \xi) \leq \operatorname{Fg}-\operatorname{cl}(\gamma) \wedge \operatorname{Fg}-\operatorname{cl}(\xi)$.

(2) $\operatorname{Fg}-\operatorname{int}(\gamma) \vee \operatorname{Fg}-\operatorname{int}(\xi) \leq \operatorname{Fg}-\operatorname{int}(\gamma \vee \xi)$.

\section{FUZZY GR-OPEN SETS AND THEIR PROPERTIES}

We introduce fuzzy GR-open sets and investigate some of their relationships between existed classes.

Definition 3.1. A fuzzy subset $\gamma$ of fuzzy space $X$ is called Fuzzy Generalized Regular open (briefly, fuzzy GR-open) set if $\gamma=\operatorname{Fint}(\mathrm{Fg}-\mathrm{cl}(\gamma))$. We denote the class of fuzzy GR-sets as $\operatorname{FGRO}(\mathrm{X})$.

Firstly we have to prove the existence of new fuzzy class in fuzzy topological spaces.

Theorem 3.1. Every fuzzy regular open set is fuzzy GR-open set, but not conversely.

Proof. Let $\gamma$ be a fuzzy regular open set in X. We know that $\gamma \leq \operatorname{Fg}-\operatorname{cl}(\gamma) \leq \operatorname{Fcl}(\gamma)$ that is $\operatorname{Fint}(\gamma) \leq \operatorname{Fint}(\operatorname{Fg} \operatorname{cl}(\gamma)) \leq \operatorname{Fint}(\operatorname{cl}(\gamma))$. As $\gamma$ is fuzzy regular open, $\gamma=\operatorname{Fint}(\operatorname{cl}(\gamma))$ and $\operatorname{Fint}(\gamma)=\gamma$. Hence $\gamma \leq \operatorname{Fint}(\operatorname{Fg}-\operatorname{cl}(\gamma)) \leq \operatorname{Fint}(\operatorname{Fcl}(\gamma))=\gamma$, Thus $\operatorname{Fint}(\operatorname{Fg}-\operatorname{cl}(\gamma))=\gamma$. Therefore $\gamma$ is fuzzy GR-open in $\mathrm{X}$.

Example 3.1. Let $\mathrm{X}=\{\mathrm{x}, \mathrm{y}, \mathrm{z}, \mathrm{w}\} ; \mathrm{I}=[0,1]$ and the function $\alpha, \beta, \gamma, \delta, \mathrm{s}, \quad \xi, \mathrm{q}$, $\phi, \varsigma, \kappa, \psi, \lambda: \mathrm{X} \rightarrow \mathrm{I}$ be defined as $\alpha(\mathrm{x})=\{(\mathrm{x}, 1)\}, \beta(\mathrm{x})=\{(\mathrm{y}, 2)\}, \xi(\mathrm{x})=\{(\mathrm{z}$, $3)\}, \quad \kappa(\mathrm{x})=\{(\mathrm{w}, 4)\}, \quad \gamma(\mathrm{x})=\{(\mathrm{x}, 1), \quad(\mathrm{y}, 2)\}, \delta(\mathrm{x})=\{(\mathrm{y}, 2), \quad(\mathrm{z}, 3)\}, \quad \mathrm{q}(\mathrm{x})=\{(\mathrm{z}, 3)$, $(\mathrm{w}, 4)\}, \quad s(\mathrm{x})=\{(\mathrm{x}, 1),(\mathrm{w}, 4)\}, \mathrm{s}(\mathrm{x})=\{(\mathrm{x}, 1),(\mathrm{y}, 2),(\mathrm{z}, 3)\}, \lambda(\mathrm{x})=\{(\mathrm{x}, 1),(\mathrm{z}, 3)$, $(\mathrm{w}, 4)\}$ and $\psi(\mathrm{x})=\{(\mathrm{y}, 2),(\mathrm{z}, 3),(\mathrm{w}, 4)\}$. Now $\tau=\left\{\begin{array}{lllll}0, & \alpha, \quad \beta, & \delta, & \mathrm{s}\end{array}\right.$, then fuzzy sets $\beta, \gamma$ are fuzzy GR-open sets but not fuzzy regular open sets in $\mathrm{X}$.

Theorem 3.2. Every fuzzy GR-open set is fuzzy open set, but converse is not true.

Proof. Let $\gamma$ be a fuzzy GR-open set in $\mathrm{X}$. That is $\gamma=\operatorname{Fint}(\mathrm{Fg}-\mathrm{cl}(\gamma))$. As fuzzy interior of any subset of $\mathrm{X}$ is fuzzy open set, therefore $\gamma$ is a fuzzy open in $\mathrm{X}$.

Example 3.2. From Example 3.1, the set $\mathrm{s}$ is fuzzy open set but not fuzzy GR-open in $X$ Remark 3.1. From Theorem 3.2, we know that every fuzzy GR-open set is a fuzzy open set but not conversely. Also from Azad K.K., every fuzzy open set is fuzzy semiopen set but not conversely. Hence every fuzzy GR-open set is a fuzzy semiopen set but not conversely.

Remark 3.2. From Theorem 3.2, we know that every fuzzy GR-open set is a fuzzy open set but not conversely. Also from Balasubramanian we know that every fuzzy open set is fuzzy g-open but not conversely. Hence every fuzzy GR-open set is a fuzzy g-open set but not conversely.

Remark 3.3. The following example shows that fuzzy GR-open sets are independent of fuzzy $\pi$ open sets, fuzzy $\delta$-open sets and fuzzy regular semiopen sets.

Example 3.3. Let $\mathrm{X}=\mathrm{Y}=\{\mathrm{a}, \mathrm{b}, \mathrm{c}, \mathrm{d}, \mathrm{e}\} ; \mathrm{I}=[0,1]$ and the function $\alpha, \mathrm{s}, \beta, \gamma$, $\xi, \psi, \lambda, \delta, \kappa, \mathrm{q}: \mathrm{X} \rightarrow \mathrm{I}$ be defined as $\alpha(\mathrm{x})=\{(\mathrm{a}, 1)\}, \mathrm{s}(\mathrm{x})=\{(\mathrm{a}, 5)\}, \beta(\mathrm{x})=\{(\mathrm{a}$, $1), \quad(\mathrm{b}, 2)\}, \quad \gamma(\mathrm{x})=\{(\mathrm{b}, 2), \quad(\mathrm{c}, 3)\}, \quad \xi(\mathrm{x})=\{(\mathrm{d}, 4), \quad(\mathrm{e}, 5)\}, \psi(\mathrm{x})=\{(\mathrm{a}, 1),(\mathrm{b}, 2),(\mathrm{c}$, $3)\}, \lambda(\mathrm{x})=\{(\mathrm{a}, 1), \quad(\mathrm{d}, 4), \quad(\mathrm{e}, 5)\}, \delta(\mathrm{x})=\{(\mathrm{b}, 2), \quad(\mathrm{c}, 3), \quad(\mathrm{e}, 5)\}, \kappa(\mathrm{x})=\{(\mathrm{a}, \quad 1), \quad(\mathrm{b}$, 2), (c, 3), (d, 4) $\}$, and $\mathrm{s}(\mathrm{x})=\{(\mathrm{b}, 2),(\mathrm{c}, 3),(\mathrm{d}, 4),(\mathrm{e}, 5)\}$, with fuzzy topology $\tau=$ $\{0, \quad 1, \alpha, \beta, \quad \gamma, \psi, \kappa\}$. Then

(1) fuzzy closed sets in $\mathrm{X}$ are $0,1, \mathrm{~s}, \xi, \lambda, \delta, \mathrm{q}$.

(2) fuzzy GR-open sets in $\mathrm{X}$ are $0,1, \alpha, \beta, \gamma, \psi$.

(3) fuzzy $\pi$-open sets in $\mathrm{X}$ are $0,1, \beta, \gamma, \kappa$. 
Research Article

(4) fuzzy $\delta$-open sets in $\mathrm{X}$ are $0,1, \beta, \gamma, \kappa$.

(5) fuzzy regular semiopen sets in $\mathrm{X}$ are $0,1, \quad \beta, \gamma, \lambda, \delta$.

Theorem 3.3. Fuzzy intersection of two fuzzy GR-open sets is a fuzzy GR-open set in fuzzy topological spaces.

Proof. Let $\gamma$ and $\xi$ be two fuzzy GR-open sets in space $\mathrm{X}$. To prove that $\gamma \wedge \xi=$ $\operatorname{Fint}(\mathrm{Fg}-\operatorname{cl}(\gamma \wedge \xi))$. As $\gamma$ and $\xi$ are fuzzy GR-open sets in $\mathrm{X}, \quad \gamma=\operatorname{Fint}(\operatorname{Fg}-\mathrm{cl}(\gamma)), \quad \xi=$ $\operatorname{Fint}(\operatorname{Fg}-\mathrm{cl}(\xi))$. We know that $\gamma \wedge \xi \leq \gamma, \operatorname{Fg}-\mathrm{cl}(\gamma \wedge \xi) \leq \operatorname{Fg}-\mathrm{cl}(\gamma)$ also $\gamma \wedge \xi \leq \xi$, Fg$\operatorname{cl}(\gamma \wedge \xi) \leq \operatorname{Fg}-\operatorname{cl}(\xi)$. Which implies $\operatorname{Fint}(\operatorname{Fg}-\operatorname{cl}(\gamma \wedge \xi)) \leq \operatorname{Fint}(\operatorname{Fg}-\operatorname{cl}(\gamma))$ and $\operatorname{Fint}(\operatorname{Fg}-\operatorname{cl}(\gamma \wedge \xi))$ $\leq \operatorname{Fint}(\operatorname{Fg}-\mathrm{cl}(\xi))$. This implies $\operatorname{Fint}(\mathrm{g}-\mathrm{cl}(\gamma \wedge \xi)) \wedge \operatorname{Fint}(\operatorname{Fg}-\mathrm{cl}(\gamma \wedge \xi)) \leq \operatorname{Fint}(\mathrm{Fg}-\mathrm{cl}(\gamma)) \wedge \operatorname{Fint}(\mathrm{Fg}-$ $\operatorname{cl}(\xi))$. That is $\operatorname{Fint}(\operatorname{Fg}-\operatorname{cl}(\gamma \wedge \xi)) \leq \operatorname{Fint}(\operatorname{Fg}-\mathrm{cl}(\gamma)) \wedge \operatorname{Fint}(\operatorname{Fg}-\operatorname{cl}(\xi))=\gamma \wedge \xi \ldots(\mathrm{i}) . \quad \gamma \wedge \xi=$ $\operatorname{Fint}(\gamma) \wedge \operatorname{Fint}(\xi)=\operatorname{Fint}(\gamma \wedge \xi)[\gamma=\operatorname{Fint}(\gamma)$ and $\xi=\operatorname{Fint}(\xi)$ because of if $\gamma$ and $\xi$ are fuzzy open sets, then every fuzzy GR-open set is fuzzy open in X] $\operatorname{Fint}(\gamma \wedge \xi) \leq$ $\operatorname{Fint}(F g-\operatorname{cl}(\gamma \wedge \xi)) . \quad \gamma \wedge \xi \leq \operatorname{Fint}(\operatorname{Fg}-\operatorname{cl}(\gamma \wedge \xi)) \ldots($ ii). From (i) and (ii), $\gamma \wedge \xi=\operatorname{Fint}(\mathrm{Fg}-$ $\operatorname{cl}(\gamma \wedge \xi))$. Hence $\gamma \wedge \xi$ is fuzzy GR-open set in $\mathrm{X}$.

Remark 3.4. The fuzzy union of two fuzzy GR-open sets is generally not a fuzzy GR-open set in fuzzy topological spaces.

Example 3.4. From Example 3.1, $\gamma$ and $\delta$ are fuzzy GR-open sets but $\gamma \vee \delta$ is not fuzzy GR-open set.

Theorem 3.4. If $\gamma$ is a fuzzy GR-open then $\operatorname{Fint}(\gamma)=\gamma$.

Proof. Let $\gamma$ is fuzzy GR-open. To prove $\operatorname{Fint}(\gamma)=\gamma$. We know that every fuzzy GR-open set is fuzzy open, that is $\gamma$ is fuzzy open set then $\operatorname{Fint}(\gamma)=\gamma$. The converse of above theorem need not be true.

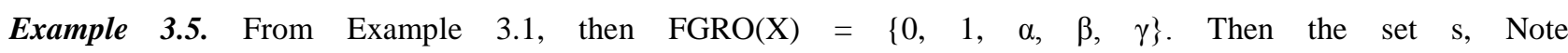
that Fint(s) is not a fuzzy GR-open set, but it is fuzzy open set.

Theorem 3.5. If $\gamma$ is fuzzy g-closed and fuzzy open in $\mathrm{X}$, then $\gamma$ is fuzzy GR-open in $\mathrm{X}$. Proof. Let $\gamma$ is fuzzy g-closed and fuzzy open in X. To prove that $\gamma=\operatorname{Fint}(\operatorname{Fg}$-cl $(\gamma))$. Now $\operatorname{Fg}-\operatorname{cl}(\gamma)=\gamma$, because $\gamma$ is fuzzy $\mathrm{g}$-open set. As $\operatorname{Fint}(\operatorname{Fg}-\operatorname{cl}(\gamma))=\operatorname{Fint}(\gamma)$ this implies $\operatorname{Fint}(\mathrm{Fg}-$ $\operatorname{cl}(\gamma))=\gamma$, because $\gamma$ is fuzzy open set. Then $\gamma$ is fuzzy GR-open in X.

Remark 3.5. Complement of a fuzzy GR-open set need not be fuzzy GR-open set.

Example 3.6. From Example 3.1, $\gamma$ is a fuzzy GR-open set. But $1-\gamma=\xi$ is not a fuzzy GR-open set.

\section{FUZZY GR-OPEN SETS AND THEIR PROPERTIES}

Definition 4.1. A fuzzy subset $\gamma$ of space $X$ is called Fuzzy Generalized Regular closed (briefly, fuzzy GR-closed) set if $1-\gamma$ is fuzzy GR-closed in $\mathrm{X}$. Then its family is denoted as $\operatorname{FGRC}(\mathrm{X})$. This new class of sets properly lies between the class of fuzzy regular closed sets and the class of fuzzy closed sets.

Theorem 4.1. A fuzzy subset $\gamma$ of $X$ is fuzzy GR-closed if and only if $\gamma=\operatorname{Fcl}(\mathrm{Fg}$-int $(\gamma))$.

Theorem 4.2. Every fuzzy regular closed set is fuzzy GR-closed set, but not conversely.

Example 4.1. From Example 3.1, set $\mathrm{q}$ and $\lambda$ are fuzzy GR-closed sets but not fuzzy regular closed in $\mathrm{X}$.

Theorem 4.3. Every fuzzy GR-closed set is fuzzy closed set, but converse is not true.

Example 4.2. From Example 3.1, the set $\kappa$ is closed set but not fuzzy GR-closed set in $X$. Remark 4.1. From Theorem 4.3, we have, every fuzzy GR-closed set is a fuzzy closed set but not conversely. Also from Azad, every fuzzy closed set is fuzzy semiclosed set but not conversely. Hence every fuzzy GR-closed set is a fuzzy semiclosed set but not conversely.

Remark 4.2. From Theorem 4.3, we know that every fuzzy GR-closed set is a fuzzy closed set but not conversely. Also from Balasubramanian, every fuzzy closed set is fuzzy g-closed but not conversely. Hence every fuzzy GR-closed set is a fuzzy g-closed set but not conversely.

Remark 4.3. The following example shows that fuzzy GR-closed sets are independent of fuzzy $\pi$-closed sets, fuzzy $\delta$-closed sets and fuzzy regular semiopen (=fuzzy regular semiclosed) sets. 
Research Article

Theorem 4.4. Fuzzy union of two fuzzy GR-closed sets is a fuzzy GR-closed set in fuzzy topological spaces.

Proof. Let $\gamma$ and $\xi$ be two fuzzy GR-closed sets in X. As $\gamma$ and $\xi$ are fuzzy GR-closed sets in $\mathrm{X}, \quad \gamma=\operatorname{Fcl}(\mathrm{Fg}-\operatorname{int}(\gamma)), \quad \xi=\operatorname{Fcl}(\mathrm{Fg}$-int $(\xi))$. We know that $\gamma \leq \mathrm{M} \vee \xi, \mathrm{Fg}$-int $(\gamma) \leq$ $\operatorname{Fg}$-int $(\gamma \quad \vee \quad \xi)$ also $\xi \leq \gamma \vee \xi$, $F$ g-int $(\xi) \leq F g-i n t(\gamma \vee \xi)$. Which $\operatorname{implies} \operatorname{Fcl}(\operatorname{Fg}$-int $(\gamma)) \leq$ $\operatorname{Fcl}(\operatorname{Fg}-i n t(\gamma \quad \vee \quad \xi))$ and $\operatorname{Fcl}(F g-i n t(\xi) \leq \operatorname{Fcl}(F g-i n t(\gamma \quad \vee \quad \xi))$. This implies $\operatorname{Fcl}(F g-i n t(\gamma)) \quad \vee \quad F c l(F g-$ $\operatorname{int}(\xi)) \leq \operatorname{Fcl}(F g-\operatorname{int}(\gamma \quad \vee \quad \xi)) \vee \operatorname{Fcl}(F g-i n t(\gamma \quad \vee \quad \xi))$. That is $\operatorname{Fcl}(\operatorname{Fg}-\operatorname{int}(\gamma)) \vee F c l(F g-i n t(\xi)) \leq$ $\operatorname{Fcl}(\operatorname{Fg}-i n t(\gamma \vee \xi)) \ldots(i) . \quad \gamma \vee \xi=\operatorname{Fcl}(\gamma) \vee \operatorname{Fcl}(\xi)=\operatorname{Fcl}(\gamma \vee \xi) \quad[\gamma=\operatorname{Fcl}(\gamma)$ and $\xi=\operatorname{Fcl}(\xi)$ and $\gamma, \xi$ are fuzzy closed sets, because every fuzzy GR-closed is fuzzy closed set] Fcl $(\gamma$ $\vee \xi) \leq \operatorname{Fcl}(\operatorname{Fg}-i n t(\gamma \vee \xi))$ i.e. $\gamma \vee \xi \leq \operatorname{Fcl}(\operatorname{Fg}-i n t(\gamma \vee \xi))$..(ii). From(i) and (ii), $\gamma \quad \vee \quad \xi$ $=\operatorname{Fcl}(\operatorname{Fg}$-int $(\gamma \vee \xi))$. Hence $\gamma \quad \vee \quad \xi$ is fuzzy GR-closed set in $\mathrm{X}$. Hence $\gamma \quad \vee \quad \xi \quad$ is fuzzy GR-closed in $\mathrm{X}$.

Remark 4.4. The fuzzy intersection of two fuzzy GR-closed sets in fuzzy topological spaces is generally, not a fuzzy GR-closed set.

Theorem 4.5. If $\gamma$ is a fuzzy GR-closed if and only if $\operatorname{Fcl}(\gamma)=\gamma$.

Proof. If $\gamma$ is fuzzy GR-closed. To prove $\operatorname{Fcl}(\gamma)=\gamma$. We know that every fuzzy GR-closed set is fuzzy closed set i.e. $\gamma$ is fuzzy closed then $\operatorname{Fcl}(\gamma)=\gamma$. But converse is not true.

Example 4.3. Let $\mathrm{X}=\{\mathrm{x}, \mathrm{y}, \mathrm{z}, \mathrm{w}\} ; \mathrm{I}=[0,1]$ and the function $\alpha, \beta, \xi, \gamma, \mathrm{s}, \mathrm{q}, \phi$, $\lambda, \quad \psi: \mathrm{X} \rightarrow \mathrm{I}$ be defined as $\alpha(\mathrm{x})=\{(\mathrm{x}, 1)\}, \quad \beta(\mathrm{x})=\{(\mathrm{y}, 2)\}, \quad \xi(\mathrm{x})=\{(\mathrm{w}, 4)\}, \gamma(\mathrm{x})$ $=\{(\mathrm{x}, 1),(\mathrm{y}, 2)\}, \mathrm{q}(\mathrm{x})=\{(\mathrm{z}, 3),(\mathrm{w}, 4)\}, \mathrm{s}(\mathrm{x})=\{(\mathrm{x}, 1),(\mathrm{y}, 2), \quad(\mathrm{z}, 3)\}, \lambda(\mathrm{x})=\{(\mathrm{x}$, $1),(\mathrm{z}, 3),(\mathrm{w}, 4)\}$ and $\psi(\mathrm{x})=\{(\mathrm{y}, 2),(\mathrm{z}, 3),(\mathrm{w}, 4)\}$. Now $\tau=\{0,1, \alpha, \beta, \gamma, \mathrm{s}\}$. $\operatorname{FGRC}(X)=\{0,1, q, \lambda, \psi\}$. Then the set $\xi$. Note that $\operatorname{Fcl}(\xi)=\xi$ is not a fuzzy GRclosed set, but it is a fuzzy closed set of $\mathrm{X}$.

Theorem 4.6. If $\gamma$ is fuzzy g-open and fuzzy closed in $X$, then $\gamma$ is fuzzy GR-closed set in $\mathrm{X}$.

Proof. Let $\gamma$ is fuzzy g-open and fuzzy closed set in $\mathrm{X}$. To prove that $\gamma$ is fuzzy GRclosed set i.e. to prove $\gamma=\operatorname{Fcl}(\mathrm{Fg}$-int $(\gamma))$. Now $\operatorname{Fg}$-int $(\gamma)=\gamma$, because $\gamma$ is fuzzy g-open set. As $\operatorname{Fcl}(\operatorname{Fg}$-int $(\gamma))=\operatorname{Fcl}(\gamma)$ this implies $\operatorname{Fcl}(\operatorname{Fg}$-int $(\gamma))=\gamma$, because $\gamma$ is fuzzy closed set. Then $\gamma$ is fuzzy GR-closed set in $\mathrm{X}$.

\section{FUZZY GR-NEIGHBOURHOOD AND FUZZY GR-INTEREIOR}

Definition 5.1. (i) Let $X$ be a fuzzy topological space and $x \in X$, A fuzzy subset $\gamma$ of $X$ is said to be a fuzzy GR-neighbourhood (briefly, FGR-nhd) of $x$ if and only if there exists a fuzzy GR-open set $\psi$ such that $\mathrm{x} \in \psi \leq \gamma$.

(ii) The collection of all fuzzy GR-neighbourhood of $x \in X$ is fuzzy GR-neighbourhood system at $\mathrm{x}$ and is denoted by FGR-N (x).

Analogous to fuzzy interior in a fuzzy space $X$, we define fuzzy GR-interior in a fuzzy space $\mathrm{X}$ as follows.

Definition 5.2. Let $\gamma$ be a fuzzy subset of $X$. A point $x \in \gamma$ is said to be fuzzy GRinterior points of $\gamma$ is called the fuzzy GR-interior of $\gamma$ and is denoted as FGR-int $(\gamma)$. point of $\gamma$ if and only if $X$ is a fuzzy GR-neighborhood of $x$. The set of all fuzzy GRinterior

Theorem 5.1. If $\gamma$ is a fuzzy subset of $X$, then FGR-int $(\gamma)=\vee\{\psi \quad: \quad \psi$ is fuzzy GRopen set, $\psi \leq \gamma\}$.

Proof. Let $\gamma$ be a fuzzy subset of $X . \quad x \in$ FGR-int $(\gamma)$ implies that $x$ is a fuzzy GRinterior point of $\mathrm{X}$ i.e. $\gamma$ is a FGR-nhd of point $\mathrm{x}$. Then there exists a fuzzy GR-open set $\psi$ such that $\mathrm{x} \in \psi \leq \gamma$ implies that $\mathrm{x} \in \mathrm{V}\{\psi: \psi$ is fuzzy GR-open set, $\psi \leq \gamma\}$. Hence FGR-int $(\gamma)=V\{\psi: \psi$ is fuzzy GR-open set, $\psi \leq \gamma\}$.

Theorem 5.2. Let $\mathrm{X}$ be a fuzzy topological space and $\gamma \leq \mathrm{X}$, then show that $\gamma$ is fuzzy GR-open set if and only if $\operatorname{FGR}-\operatorname{int}(\gamma)=\gamma$.

Proof. Let $\gamma$ be a fuzzy GR-open set in $\mathrm{X}$. Then clearly the biggest fuzzy GR-open set contained in $\gamma$, is itself $\gamma$. Hence FGR-int $(\gamma)=\gamma$. 
Research Article

Conversely, suppose that $\gamma \leq \mathrm{X}$ and FGR-int $(\gamma)=\gamma$. Since FGR-int $(\gamma)$ is a fuzzy GR-open set in $\mathrm{X}$, it follows that $\gamma$ is a fuzzy GR-open set in $\mathrm{X}$.

Theorem 5.3. Let $\gamma$ and $\xi$ are fuzzy subset of $\mathrm{X}$. Then

(1) FGR-int $(X)=1$ and FGR-int $(\varnothing)=0$.

(2) $\operatorname{FGR}-\operatorname{int}(\gamma) \leq \gamma$.

(3) If $\xi$ is any fuzzy GR-open set contained in $\gamma$, then $\xi \leq$ FGR-int $(\gamma)$.

(4) If $\gamma \leq \xi$, then FGR-int $(\gamma) \leq \operatorname{FGR}-\operatorname{int}(\xi)$.

(5) FGR-int(FGR-int $(\gamma)=$ FGR-int $(\gamma)$.

Proof. (1) Since $X$ and $\varnothing$ are fuzzy GR-open sets, by Theorem 5.1, FGR-int $(X)=\vee\{\psi$ : $\psi$ is fuzzy GR-open set, $\psi \leq \mathrm{X}\}=\mathrm{X} \vee\{$ all fuzzy GR-open sets $\}=\mathrm{X}$. That is FGR-int $(X)=X=1$. Since $\varnothing$ is the only fuzzy GR-open set contained in $\varnothing$, FGR-int(Ø) $=\varnothing=0$.

(2) Let $\mathrm{x} \in$ FGR-int $(\gamma)$ implies that $\mathrm{x}$ is a fuzzy GR-interior point of $\gamma$. That is $\gamma$ is a

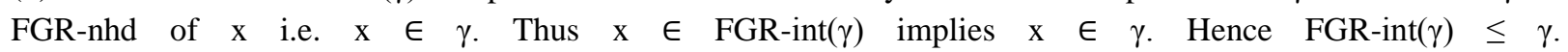

(3) Let $\xi$ be any fuzzy GR-open set such that $\xi \leq \gamma$. Let $\mathrm{x} \in \xi$. Since $\xi$ is a fuzzy GR-open set contained in $\gamma, \mathrm{x}$ is a fuzzy GR-interior point of $\gamma$. That is $\mathrm{x} \in$ FGR-int $(\gamma)$. Hence $\xi \leq$ FGR-int $(\gamma)$.

(4) Let $\gamma$ and $\xi$ be subsets of $\mathrm{X}$ such that $\gamma \leq \xi$. Let $\mathrm{x} \in$ FGR-int $(\gamma)$. Since FGR-int $(\gamma)$ $\leq \gamma$ and $\gamma \leq \xi$, we have FGR-int $(\gamma) \leq \xi$. Now FGR-int $(\gamma)$ is a fuzzy GR-open set and FGR-int $(\xi)$ is the biggest fuzzy GR-open set contained in $\xi$, we have to find FGR-int $(\gamma) \leq$ FGR-int $(\xi)$.

(5) Since FGR-int $(\gamma)$ is a fuzzy GR-open set in $\mathrm{X}$, it follows that FGR-int(FGR-int $(\gamma))=$ FGR$\operatorname{int}(\gamma)$.

Theorem 5.4. If $\gamma$ and $\xi$ are fuzzy subsets of $X$, then FGR-int $(\gamma) \vee$ FGR-int $(\xi) \leq$ FGR-int $(\gamma$ $\vee \xi)$.

Proof. We know that $\gamma \leq \gamma \vee \xi$ and $\xi \leq \gamma \vee \xi$. We have, by Theorem 5.5(iv), FGR$\operatorname{int}(\gamma) \leq \operatorname{FGR}-\operatorname{int}(\gamma \vee \xi)$ and $\operatorname{FGR}-\operatorname{int}(\xi) \leq \operatorname{FGR}-\operatorname{int}(\gamma \vee \xi)$. This implies FGR-int $(\gamma) \vee$ FGR$\operatorname{int}(\xi) \leq$ FGR-int $(\gamma \vee \xi)$.

Theorem 5.5. Let $\gamma$ and $\xi \quad$ are subsets of $X$, then FGR-int $(\gamma) \vee \quad$ FGR-int $(\xi)=$ FGR-int $(\gamma \quad \vee \quad \xi)$. Proof. We know that $\gamma \vee \xi \leq \gamma$ and $\gamma \vee \xi \leq \xi$. We have, by Theorem 5.3(iv), FGR$\operatorname{int}(\gamma \vee \xi) \leq \operatorname{FGR}-\operatorname{int}(\gamma)$ and $\operatorname{FGR}-\operatorname{int}(\gamma \vee \xi) \leq \operatorname{FGR}-\operatorname{int}(\xi)$. This implies $\operatorname{FGR}-\operatorname{int}(\gamma \vee \xi) \leq$ FGR-int $(\gamma) \vee \quad$ FGR-int $(\xi)$...(i). Again, let $x \in$ FGR-int $(\gamma) \vee \quad F G R-i n t(\xi)$. Then $\quad x \in$ FGR-int $(\gamma)$ and $\quad x$ $\in$ FGR-int $(\xi)$. Hence $x$ is an fuzzy interior point of each of fuzzy sets $\gamma$ and $\xi$. It follows that $\gamma$ and $\xi$ are FGR-nhd of $\mathrm{x}$, so that their fuzzy intersection $\gamma \vee{ }$ is also a FGR-nhd of $\mathrm{x}$. Hence $\mathrm{x} \in \mathrm{FGR}-\operatorname{int}(\gamma \quad \vee \quad \xi)$. Thus $\mathrm{x} \in \mathrm{FGR}-\operatorname{int}(\gamma) \vee$ FGR-int $(\xi)$ implies that $\mathrm{x}$ $\in$ FGR-int $(\gamma \vee \xi)$. Therefore FGR-int $(\gamma) \vee$ FGR-int $(\xi) \leq$ FGR-int $(\gamma \vee \xi)$...(ii). From (i) and (ii), we get FGR-int $(\gamma) \vee \quad$ FGR-int $(\xi)=$ FGR-int $(\gamma \quad \vee \quad \xi)$.

\section{FUZZY GR-CLOSURE AND THEIR PROPERTIES}

Using the fuzzy GR-closed sets we can introduce the concept of fuzzy GR-closure operator in fuzzy topological spaces.

Definition 6.1. Let $\gamma$ be a fuzzy subset of X. We define the fuzzy GR-closure of $\gamma$ by fuzzy intersection of all fuzzy GR-closed sets containing $\gamma$. Mathematically, FGR-cl $(\gamma)=\wedge\{\xi$ : to be the $\gamma \leq \xi \in \operatorname{FGRC}(X)\}$.

Theorem 6.1. Let $\mathrm{X}$ be any fuzzy topological space and $\gamma \leq \mathrm{X}$, then show that $\gamma$ is fuzzy GR closed set if and only if $\operatorname{FGR}-\operatorname{cl}(\gamma)=\gamma$.

Proof. Let $\gamma$ be a fuzzy GR-closed set in X. Then clearly the smallest fuzzy GR-closed set contained in $\gamma$, is itself $\gamma$. Hence $\operatorname{FGR}-\operatorname{cl}(\gamma)=\gamma$.

Conversely, suppose that $\gamma \leq \mathrm{X}$ and $\operatorname{FGR}-\mathrm{cl}(\gamma)=\gamma$. Since FGR-cl $(\gamma)$ is a fuzzy GR-open set in $\gamma$, it follows that $\gamma$ is a fuzzy GR-closed set in $\mathrm{X}$.

Theorem 6.2. Let $\gamma$ and $\xi$ are subset of $X$. Then

(1) $\operatorname{FGR}-\operatorname{cl}(X)=1$ and FGR-cl(Ø) $=0$. 
Research Article

(2) $\gamma \leq$ FGR-cl $(\gamma)$.

(3) If $\xi$ is any fuzzy GR-closed set contained in $\gamma$, then FGR-cl $(\gamma) \leq \xi$.

(4) If $\gamma \leq \xi$, then FGR-cl $(\gamma) \leq$ FGR-cl $(\xi)$.

(5) $\mathrm{R}-\mathrm{cl}(\mathrm{GR}-\mathrm{cl}(\xi))=\mathrm{GR}-\mathrm{cl}(\xi)$.

Proof. (1) Obviously.

(2) By the definition of fuzzy GR-closure of $\gamma$, it is obvious that $\gamma \leq$ FGR-cl $(\gamma)$.

(3) Let $\xi$ be any fuzzy GR-closed set containing $\gamma$. Since FGR-cl $(\gamma)$ is the fuzzy intersection of all fuzzy GR-closed sets containing $\gamma$ i.e FGR-cl $(\gamma)$ is contained in every fuzzy GR-closed set containing $\gamma$. Hence FGR-cl $(\gamma) \leq \xi$.

(4) Let $\gamma$ and $\xi$ are fuzzy subsets of $\mathrm{X}$ such that $\gamma \leq \xi$. By the definition of fuzzy GRclosure, FGR-cl $(\xi)=\wedge\{\psi: \xi \leq \psi \in \operatorname{FGRC}(\mathrm{X})\}$. If $\xi \leq \psi \in \operatorname{FGRC}(\mathrm{X})$, then $\mathrm{FGR}-\mathrm{cl}(\xi) \leq$ $\psi$. Since $\gamma \leq \xi, \gamma \leq \xi \leq \psi \in \operatorname{FGRC}(\mathrm{X})$, we have $\operatorname{FGR}-\operatorname{cl}(\gamma) \leq \psi$. Therefore FGR-cl $(\gamma) \leq$ $\wedge\{\psi: \xi \leq \psi \in \operatorname{FGRC}(\mathrm{X})\}=\mathrm{FGR}-\mathrm{cl}(\mathrm{X})$. That is $\mathrm{FGR}-\mathrm{cl}(\gamma) \leq \mathrm{FGR}-\mathrm{cl}(\xi)$.

(5) Since FGR-cl $(\gamma)$ is a fuzzy GR-closed set in X. It follows that FGR-cl(FGR-cl(X)) = X. Theorem 6.3. Let $\gamma$ and $\xi$ are fuzzy subsets of $X$, then FGR-cl $(\gamma \vee \xi)=$ FGR-cl $(\gamma) \vee$ FGR$\mathrm{cl}(\xi)$. Proof. Let $\gamma$ and $\xi$ are fuzzy subsets of X. Clearly $\gamma \leq \gamma \vee \xi$ and $\xi \leq \gamma \vee \xi$. We have by the Theorem 6.2(iv), FGR-cl $(\gamma) \leq$ FGR-cl $(\gamma \vee \xi)$ and FGR-cl $(\xi) \leq$ FGR-cl $(\gamma \vee \vee$ $\xi)$. This implies FGR-cl $(\gamma) \vee$ FGR-cl $(\xi) \leq$ FGR-cl $(\gamma \vee \xi) \ldots(i)$. Now to prove that FGR-cl $(\gamma \vee \vee$

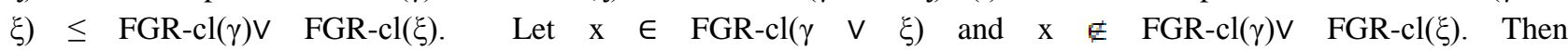
there exists fuzzy GR-closed sets $\gamma_{1}$ and $\xi_{1}$ with $\gamma \leq \gamma_{1}, \xi \leq \xi_{1}$ and $\mathrm{x} \notin \gamma_{1} \vee \xi_{1}$. We have $\gamma \vee \xi \leq \gamma_{1} \vee \xi_{1}$ and $\gamma_{1} \vee \xi_{1}$ is a fuzzy GR-closed set by Theorem 6.2, such that $\mathrm{x} \notin \gamma_{1} \quad \vee \quad \xi_{1}$. Thus $\mathrm{x} \notin \operatorname{FGR}-\mathrm{cl}(\gamma \quad \vee \quad \xi)$ which is contradiction to $\mathrm{x} \in \mathrm{FGR}-\mathrm{cl}(\gamma$ $\vee \xi)$. Hence FGR-cl $(\gamma \quad \vee \quad \xi) \leq$ FGR-cl $(\gamma) \vee$ FGR-cl $(\xi) \ldots$ (ii). From (i) and (ii), we have FGR$\operatorname{cl}(\gamma \quad \vee \quad \xi)=\operatorname{FGR}-\operatorname{cl}(\gamma) \vee \quad$ FGR-cl $(\xi)$.

Theorem 6.4. Let $\gamma$ and $\xi$ are fuzzy subsets of $X$, then FGR-cl $(\gamma \wedge \xi) \leq$ FGR-cl $(\gamma) \wedge$ FGR-cl $(\xi)$.

Proof. Let $\gamma$ and $\xi$ are fuzzy subsets of $\mathrm{X}$. Clearly $\gamma \wedge \xi \leq \gamma$ and $\gamma \wedge \xi \leq \xi$. We have, by Theorem 6.2(iv), FGR-cl $(\gamma \wedge \xi) \leq \operatorname{FGR}-\operatorname{cl}(\gamma)$ and $\operatorname{FGR}-\operatorname{cl}(\gamma \wedge \xi) \leq \operatorname{FGR}-\mathrm{cl}(\xi)$. This implies FGR-cl $(\gamma \wedge \xi) \leq$ FGR-cl $(\gamma) \wedge \operatorname{FGRcl}(\xi)$.

Remark 6.1. In general $\operatorname{FGR}-\operatorname{cl}(\gamma) \wedge$ FGR-cl $(\xi) \quad \nless \quad \operatorname{FGR}-\operatorname{cl}(\gamma \wedge \xi), \quad$ as $\quad$ seen from the following example.

Example 6.1. Consider Example, $\delta, \quad \mathrm{q}, \delta \wedge \mathrm{q}=\xi$, FGR-cl $(\delta)=\psi, \quad$ FGR-cl(q) $=\mathrm{q}$, FGR$\operatorname{cl}(\delta \wedge \mathrm{q})=\xi$ and $\operatorname{FGR}-\operatorname{cl}(\delta) \wedge \operatorname{FGR}-\mathrm{cl}(\mathrm{q})=\mathrm{q}$. Therefore $\operatorname{FGR}-\operatorname{cl}(\delta) \wedge$ FGR-cl(q) $\nless$ GR-cl $(\delta$ $\wedge q)$. Theorem 6.5. Let $\gamma$ be a fuzzy subset of $X$ and $x \in X$. Then $x \in$ FGR-cl $(\gamma)$ if and only if $\delta \wedge \gamma f=\varnothing$ for every fuzzy GR-open set $\delta$ containing $\mathrm{x}$.

Proof. Let $\mathrm{x} \in \mathrm{X}$ and $\mathrm{x} \in \operatorname{FGR}-\mathrm{cl}(\gamma)$. To prove that $\delta \wedge \gamma f=\varnothing$ for every fuzzy GRopen set $\delta$ containing $x$. Prove the results by contradiction. Suppose there exists a fuzzy GRopen set $\delta$ containing $\mathrm{x}$ such that $\delta \wedge \gamma=\varnothing$. Then $\gamma \leq 1-\delta$ and $1-\delta$ is fuzzy GR-closed set. We have FGR-cl $(\gamma) \leq 1-\delta$. This shows that $\mathrm{x} \notin$ FGR-cl $(\gamma)$, which is a contradiction. Hence $\delta \wedge \gamma f=\varnothing$ for every fuzzy GR-open set $\delta$ containing x.

Conversely, let $\delta \wedge \gamma f=\varnothing$ for every fuzzy GR-open set $\delta$ containing $\mathrm{x}$. To prove that $\mathrm{x}$ $\in$ FGR- $\operatorname{cl}(\gamma)$. We prove the result by contradiction. Suppose $\mathrm{x} \notin$ FGR-cl $(\gamma)$. Then there exists a fuzzy GR-closed subset $\xi$ containing $\gamma$ such that $\mathrm{x} \notin \xi$. Then $\mathrm{x} \in 1-\xi$ and $1-\xi$ is fuzzy GR-open set. Also $(1-\xi) \wedge \gamma=\varnothing$, which is a contradiction. Hence $\mathrm{x}$ $\in \operatorname{FGR}-\operatorname{cl}(\gamma)$.

Definition 6.2. Let $\mathrm{X}$ be fuzzy topological space and $\tau_{\mathrm{FGR}}=\left\{\delta \leq \mathrm{X}:\right.$ FGR-cl $\left.\left(\delta^{\mathrm{c}}\right)=\delta^{\mathrm{c}}\right\}$. $\tau_{\mathrm{FGR}}$ is fuzzy topology on $\mathrm{X}$.

Lemma 6.1. Let $\gamma$ be a fuzzy subset of a fuzzy space $x$. Then

(i) $1-($ FGR-int $(\gamma))=\operatorname{FGR}-\mathrm{cl}(1-\gamma)$.

(ii) $\operatorname{FGR}-\operatorname{int}(\gamma)=1-(\operatorname{FGR}-\mathrm{cl}(1-\gamma))$.

(iii) $\operatorname{FGR}-\operatorname{cl}(\gamma)=1-($ FGR-int $(1-\gamma))$. 
Research Article

Proof. Let $\mathrm{x} \in 1-(\mathrm{FGR}-\operatorname{int}(\gamma))$. Then $\mathrm{x} \in /$ FGR-int $(\gamma)$. That is every fuzzy GR-open set $\xi$ containing $\mathrm{x}$ is such that $\xi \notin \gamma$. That is every fuzzy GR-open set $\xi$ containing $\mathrm{x}$ is such that $\xi \wedge(1-\gamma) f=\varnothing$. By the Theorem, $\mathrm{x} \in$ FGR-cl( $1-\gamma)$ and therefore 1-(FGR$\operatorname{int}(\gamma)) \leq$ FGR- $\operatorname{cl}(1-\gamma)$

Conversely, let $\mathrm{x} \in$ FGR-cl(1- $-\gamma)$, Then by Theorem, every fuzzy GR-open set $\xi$ containing $\mathrm{x}$ is such that $\xi \wedge(1-\gamma) f=\varnothing$. That is every fuzzy GR-open set $\xi$ containing $\mathrm{x}$ is such that $\xi \nless \mathrm{M}$. By definition of fuzzy GR-interior of $\gamma, \mathrm{x} \notin$ FGR-int $(\gamma)$. That is $\mathrm{x} \in$ $1-($ FGR-int $(\gamma))$ and FGR-cl( $1-\gamma) \leq 1-($ FGR-int $(\gamma))$. Thus $1-($ FGR-int $(\gamma))=$ FGR-cl $(1-\gamma)$.

Follows by taking complements in (i).

Follows by replacing $\gamma$ by $1-\gamma$ in (i).

\section{FUZZY GR-CONTINUOUS MAPS AND THEIR PROPERTIES}

We introduce a new class of fuzzy maps called fuzzy Generalized Regular (briefly, fuzzy GR- continuous) maps and discuss their characterizations.

Definition 7.1. A fuzzy map $\mathrm{f}: \mathrm{X} \rightarrow \mathrm{Y}$ is said to be fuzzy Generalized Regular continuous (briefly, fuzzy GR-continuous) if the inverse image of every fuzzy closed set in $Y$ is fuzzy GR-closed set in $\mathrm{X}$.

Theorem 7.1. A fuzzy map f $: \mathrm{X} \rightarrow \mathrm{Y}$ is fuzzy GR-continuous if and only if the inverse image of a fuzzy open set in $\mathrm{Y}$ is fuzzy GR-open set in $\mathrm{X}$.

Proof. Let $\mathrm{f}: \mathrm{X} \rightarrow \mathrm{Y}$ be fuzzy GR-continuous and $\gamma$ be a fuzzy open set in $\mathrm{Y}$. Then $\gamma^{\mathrm{c}}$ is fuzzy closed set in $\mathrm{X}$. Since $\mathrm{f}$ is fuzzy GR-continuous, $\mathrm{f}-1\left(\gamma^{\mathrm{c}}\right)$ is fuzzy GR-closed in $\mathrm{X}$. But $\mathrm{f}^{-1}\left(\gamma^{\mathrm{c}}\right)=\left(\mathrm{f}^{-1}(\gamma)\right)^{\mathrm{c}}$ and so $\mathrm{f}^{-1}(\gamma)$ is fuzzy GR-open set in $\mathrm{X}$.

Conversely, assume that $\mathrm{f}-1(\gamma)$ is fuzzy GR-open set in $\mathrm{X}$ for each fuzzy open set $\gamma$ in $\mathrm{Y}$. Let $\delta$ be a fuzzy closed set in $\mathrm{Y}$ and $\mathrm{f}^{-1}\left(\delta^{\mathrm{c}}\right)$ is fuzzy GR-open set in $\mathrm{X}$. Since $\mathrm{f}^{-1}\left(\delta^{\mathrm{c}}\right)=$ $\left(\mathrm{f}^{-1}(\delta)\right)^{\mathrm{c}}$. That is $\mathrm{f}^{-1}(\delta)$ is fuzzy GR-closed in $\mathrm{X}$. Therefore $\mathrm{f}$ is fuzzy GR-continuous.

Theorem 7.2. If a fuzzy map $\mathrm{f}: \mathrm{X} \rightarrow \mathrm{Y}$ is fuzzy completely continuous, then it is fuzzy GR-continuous.

Proof. Let $\mathrm{f}: \mathrm{X} \rightarrow \mathrm{Y}$ be a fuzzy completely continuous map. Let $\psi$ be a any fuzzy closed set in $Y$. Since $f$ is fuzzy completely continuous, $f^{-1}(\psi)$ is fuzzy regular closed set in $\mathrm{X}$. By Theorem, every fuzzy regular closed set is fuzzy GR-closed set in $\mathrm{X}$. $\mathrm{f}^{-1}(\psi)$ is fuzzy GR-closed set in $X$. Therefore $f$ is fuzzy GR-continuous. But converse of the theorem need not be true.

Example 7.1. From Example 3.3, $\tau=\left\{0,1_{\mathrm{X}}, \alpha, \beta, \gamma, \delta, \mathrm{s}\right\}$ and $\sigma=\left\{0,1_{\mathrm{Y}}, \alpha, \beta, \gamma\right.$, $\mathrm{s}\}$. Let $\mathrm{f}: \mathrm{X} \rightarrow \mathrm{Y}$ be defined by $\mathrm{f}(\mathrm{x})=\mathrm{y}, \mathrm{f}(\mathrm{y})=\mathrm{z}, \mathrm{f}(\mathrm{z})=\mathrm{z}$ and $\mathrm{f}(\mathrm{w})=$ w. Then $f$ is GR-continuous but not completely continuous, as inverse image of GR-closed set $\psi$ in $\mathrm{Y}$ is $\mathrm{q}$ which is not regular closed set in $\mathrm{X}$.

Theorem 7.3. If a fuzzy map $\mathrm{f}: \mathrm{X} \rightarrow \mathrm{Y}$ is fuzzy GR-continuous, then it is fuzzy continuous. Proof. Let $\mathrm{f}: \mathrm{X} \rightarrow \mathrm{Y}$ be a fuzzy GR-continuous map. Let $\psi$ be any fuzzy closed set in $\mathrm{Y}$. Since $\mathrm{f}$ is fuzzy GR-continuous, $\mathrm{f}^{-1}(\psi)$ is fuzzy GR-closed set in $\mathrm{X}$. By Theorem, every fuzzy GR-closed set is fuzzy closed set in $X . f^{-1}(\psi)$ is in $X$. Therefore $f$ is fuzzy GR-continuous.

The converse of the above theorem need not be true.

Example 7.2. Let $\mathrm{X}=\mathrm{Y}=\{\mathrm{x}, \mathrm{y}, \mathrm{z}, \mathrm{w}\} ; \mathrm{I}=[0,1]$ and the function $\alpha, \beta, \gamma, \lambda, \psi$, $\xi, \mathrm{s}: \mathrm{X} \rightarrow \mathrm{I}$ be defined as $\alpha(\mathrm{x})=\{(\mathrm{x}, 1)\}, \beta(\mathrm{x})=\{(\mathrm{y}, 2)\}, \gamma(\mathrm{x})=\{(\mathrm{x}, 1),(\mathrm{y}, 2)\}$, $\lambda(\mathrm{x})=\{(\mathrm{y}, 2), \quad(\mathrm{z}, 3)\}, \quad \psi(\mathrm{x})=\{(\mathrm{y}, 2), \quad(\mathrm{w}, 4)\}, \quad \xi(\mathrm{x})=\{(\mathrm{z}, 3), \quad(\mathrm{w}, \quad 4)\}, \quad$ and $\quad \mathrm{s}(\mathrm{x})=$ $\{(\mathrm{x}, 1), \quad(\mathrm{y}, 2), \quad(\mathrm{z}, 3)\}$. Now $\tau=\{0,1, \alpha, \beta, \gamma, \mathrm{s}\}$. Let $\mathrm{X}=\mathrm{Y}=\{1,2,3,4\}$ be with topologies $\tau=\left\{0,1_{\mathrm{X}}, \alpha, \lambda, \mathrm{s}\right\}$ and $\sigma=\left\{0,1_{\mathrm{Y}}, \alpha, \beta, \gamma, \mathrm{s}\right\}$. Let $\mathrm{f}: \mathrm{X} \rightarrow$ $\mathrm{Y}$ be defined by $\mathrm{f}(\mathrm{x})=\mathrm{w}, \mathrm{f}(\mathrm{y})=\mathrm{z}, \mathrm{f}(\mathrm{z})=\mathrm{y}$ and $\mathrm{f}(\mathrm{w})=\mathrm{w}$. Then $\mathrm{f}$ is continuous but not GR-continuous, as inverse image of closed set $\xi$ in $\mathrm{Y}$ is $\psi$ it is not GR-closed set in $\mathrm{X}$. 
Research Article

Definition 7.2. A fuzzy map $\mathrm{f}: \mathrm{X} \rightarrow \mathrm{Y}$ is said to be fuzzy Generalized Regular open (briefly, fuzzy GR-open) if the image of every fuzzy open set in $\mathrm{X}$ is fuzzy GR-open set in $\mathrm{Y}$.

Theorem 7.4. If a fuzzy map $\mathrm{f}$ is fuzzy GR-open map, then it is fuzzy open map.

Proof. Let $\mathrm{f}: \mathrm{X} \rightarrow \mathrm{Y}$ be a fuzzy GR-open map. Let $\xi$ be any fuzzy open set in $\mathrm{X}$. Since $\mathrm{f}$ is fuzzy GR-open, $\mathrm{f}(\xi)$ is fuzzy GR-open set in Y. By Theorem, every fuzzy GRopen set is fuzzy open set in $Y$. $f(\xi)$ is fuzzy open set in $Y$. Therefore $f$ is fuzzy open map.

Definition 7.3. A fuzzy map $\mathrm{f}$ is said to be fuzzy Generalized Regular homeomorphism (briefly, fuzzy GR-homeomorphism) if the $\mathrm{f}$ is fuzzy GR-continuous and fuzzy GR-open map.

Theorem 7.5. Every fuzzy GR-homeomorphism is fuzzy homeomorphism.

Proof. Let $\mathrm{f}: \mathrm{X} \rightarrow \mathrm{Y}$ be a fuzzy GR-homeomorphism, i.e. $\mathrm{f}$ is fuzzy GR-continuous and fuzzy GR-open map. Since every fuzzy GR-continuous is fuzzy continuous map and fuzzy GRopen map is fuzzy open map. Therefore $f$ is fuzzy continuous and fuzzy open map. Hence $f$ is fuzzy GR-homeomorphism.

\section{REFERENCES}

[1] Anjana Bhattacharyya, Fuzzy Regular Generalized $\pi$-Closed Sets and Fuzzy Regular Generalized $\pi$ - Continuous Functions, Advances in Fuzzy Mathematics, 12(4), 1047-1066.

[2] Azad K.K., On fuzzy semi-continuity, fuzzy almost continuity and fuzzy weakly continuity, J. Math. Anal. Appl., 82 (1981), 14-32.

[3] Balasubramanian G. and Sundaram, P., On some generalizations of fuzzy continuous functions, Fuzzy Sets and Systems, 86 (1997), 93-100.

[4] Saziye Yuksel, Eser Gursel Caylak, Ahu Acikgoz, On Fuzzy $\delta$-I-Open Sets and Decomposition of Fuzzy $\alpha$-I-continuity, SDU Journal of Science, 5(2010), 147-153.

[5] Zahren A.K., Fuzzy reguar semiopen sets ans S-closed spaces, Amer.Math. Monthly, 2(1994), 579-586. 